\title{
Proper Orthogonal Decomposition of Atomizing Free-Surface Circular Liquid Sheet
}

\author{
Chetankumar S. Vegad¹, Nikhil A. Baraiya², Satyanarayanan R. Chakravarthy ${ }^{1}$, Amit Kumar ${ }^{1}$ \\ ${ }^{1}$ National Centre for Combustion Research and Development, and Department of Aerospace \\ Engineering, Indian Institute of Technology Madras, Chennai 600 036, India \\ ${ }^{2}$ Department of Mechanical Engineering, Sardar Vallabhbhai National Institute of \\ Technology, Surat 395 007, India \\ *Corresponding author email: vegad.cst@gmail.com
}

\begin{abstract}
The most dominant events associated with expanding thin circular liquid sheet breakup dynamics are extracted and quantified using proper orthogonal decomposition (POD). The circular liquid sheet is formed by an orthogonal impingement of a cylindrical water jet on the horizontally placed cone-disc deflector. For the first time, the atomization process of such liquid sheet is captured simultaneously in the side and top views using the high-speed volume laser-induced fluorescent (VLIF) technique. The atomization was carried out for different flow conditions in terms of jet Weber number $\left(W e_{j e t}\right)$ over a range 996-7480. The results of POD analysis of VLIF data revealed the sheet oscillation is the most dominant phenomenon at lower $W e_{j e t}$ and radial wave propagation is the most dominant feature at intermediate $W e_{j e t}$. Liquid sheet breakup is identified as the dominating phenomenon for higher flow rates (high $W e_{j e t}$ ). The change in dominancy of various phenomena with inlet flow conditions is observed from the POD.
\end{abstract}

\section{Keywords}

Circular liquid sheet, free-surface liquid sheet, POD, VLIF

\section{Introduction}

Atomization of free-surface (expanding) liquid sheets is a critical phenomenon to understand since it is connected to various applications, i.e., spray painting, combustion, irrigation system, liquid rocket, fire suppression system, coating, and other industrial treatments [1]. In the present work, the atomization of a radially expanding circular liquid sheet is captured simultaneously using the high-speed volume laser-induced fluorescent (VLIF) technique. The circular liquid sheet is produced using the convergent nozzle and deflector assembly. The cylindrical water jet impinges vertically onto a horizontally placed deflector creating the symmetric circular liquid sheet into the air. In the present work, various events leading to sheet atomization are extracted by employing the proper orthogonal decomposition (POD) tool.

POD was first introduced in 1943, and since then, it is being used in various disciplines [2]. The basic principle of POD is to explain high-dimensional processes with low-dimensional descriptions [3]. Its primary application is for data compression, image processing, and studying dynamical systems $[4,5]$. The turbulent flows are also studied using this tool $[6,7]$. The combined POD and Linear Stochastic Estimation (LSE) are used to identify the coherent structures of a low-dimension dynamical system [8]. POD is also used for the structural analysis of the liquid sheet profile $[9,10]$.

The current approach identifies the most dominant events involved in the sheet breakup process and investigates any change in dominancy with inlet flow conditions. The objective is 
achieved by performing spatial and temporal POD analysis to extract the spatial structures and frequencies of the most dominant features, respectively.

\section{Experimental Setup and Techniques}

The experimental setup consists of a convergent nozzle placed at $20 \mathrm{~mm}$ exactly above the cone-disc deflector. The nozzle orifice and deflector disc diameters are $9.5 \mathrm{~mm}$ and $25.4 \mathrm{~mm}$, respectively [11]. The included angle of the cone adopted in the present work is $90^{\circ}$. The water was supplied to the nozzle employing a centrifugal pump. The water mass flow rate was measured using a flow meter and the supply pressure was monitored by means of a static pressure transducer (accuracy, $\pm 0.25 \%$ FS). Water splashing against the deflector was collected in $2200 \mathrm{~mm}$ diameter and $500 \mathrm{~mm}$ high circular tank. The tank outlet was connected to the pump inlet for recirculation of water. The size of the tank was determined from preliminary tests in the free field. In the preliminary tests, the effect of the proximity of the wall on sheet breakup distance was determined. The outcome showed no effect on sheet breakup dynamics by keeping the obstacle $1000 \mathrm{~mm}$ away from the central axis. The deflector conedisc was mounted on the traverse system to provide vertical and horizontal motions (in three mutually perpendicular directions). Former to vary the distance between the nozzle and deflector, and latter to position it precisely below the nozzle.

Before starting any experiments to capture liquid sheet atomization, the K-factor of the convergent nozzle was measured by allowing the amount of water passed through the nozzle orifice for a given time and discharge pressure (gauge). Time and discharge pressure were recorded with the help of a stopwatch and pressure transducer, respectively. At the same time, the water was collected in a scientific beaker. Hence, the water flow rate was obtained from the collected volume of water over for a given time. Experiments were repeated at least three times for each flow rate. The following equation gives the K-factor of a nozzle,

$$
\mathrm{K}=\frac{Q_{l}}{\sqrt{p}}
$$

where $Q_{l}$ is the liquid flow rate (lpm), and $p$ is the discharge pressure (bar). The measured Kfactor of $9.5 \mathrm{~mm}$ nozzle orifice is found to be $58 \pm 1.64 \mathrm{lpm}$ bar $^{-0.5}$.

Since the water temperature was about $25^{\circ} \mathrm{C}$ while performing all the experiments, water properties taken at $25^{\circ} \mathrm{C}$ are density $\rho_{l}=997 \mathrm{~kg} \mathrm{~m}^{-3}$, dynamic viscosity $\mu_{l}=0.89 \times 10^{-3} \mathrm{~Pa} \mathrm{~s}$ [12], and surface tension of water-air interface $\sigma=71.2 \times 10^{-3} \mathrm{~N} \mathrm{~m}^{-1}$ [13]. The surface tension effect is included in terms of liquid jet Weber number $\left(W e_{j e t}\right)$. Experiments were performered by varying discharge pressure and so the liquid jet Weber number,

$$
W e_{j e t}=\frac{\rho_{l} U_{0}^{2} D_{0}}{\sigma}
$$

where, $U_{0}$ is jet velocity $(\mathrm{m} / \mathrm{s}), D_{0}$ is nozzle orifice diameter $(\mathrm{m}), \rho_{l}$ is liquid density $\left(\mathrm{m}^{3} / \mathrm{s}\right)$ and $\sigma$ is the surface tension of water $(\mathrm{N} / \mathrm{m})$ at $25^{\circ} \mathrm{C}$. Further details of the experimental setup are given in [14].

The entire event, starting from growing disturbances in terms of radial and azimuthal waves up to their breakup into droplets, was covered simultaneously in top and side view high-speed VLIF for the jet Weber number $\left(W e_{\text {jet }}\right)$ over the range $996<W e_{j e t}<7480$. The simultaneous imaging was carried out at $6 \mathrm{kHz}$ framing rate with the use of two high-speed CMOS cameras (Photron make) operated along with a $527 \mathrm{~nm}$ wavelength $\mathrm{Nd}$ :YLF laser (Litron make) at 6 $\mathrm{kHz}$ high-repetition-rate and at $6.7 \mathrm{~mJ}$ energy to excite the liquid sheet. The simultaneous triggering of both the cameras and the laser unit was possible using a high-speed controller. At the same time, the images were acquired using DaVis 8.3.0 software (LaVision make).

A Rhodamine $6 \mathrm{G}$ dye with a concentration of $0.4 \mathrm{~g} / \mathrm{l}$ of water at $6 \mathrm{kHz}$ laser pulse rate and 6.4 
$\mathrm{mJ}$ laser energy was used as a fluorescent media in all the VLIF experiments. The dyed liquid was then excited by a $40 \mathrm{~mm}$ wide cuboidal laser volume of a $527 \mathrm{~nm}$ wavelength. Also, the concentration used here would not affect the surface tension of water.

The volume optics was used to convert the $10 \mathrm{~mm}$ diameter laser beam into the cuboidal laser volume. In volume optics, a laser beam passes through $15 \mathrm{~mm} \times 15 \mathrm{~mm}$ and $15 \mathrm{~mm} \times 60 \mathrm{~mm}$ planoconcave lenses of respective $8 \mathrm{X}$ and $5 \mathrm{X}$ magnification factors to generate a laser volume by varying the distance between these lenses. When a laser volume strikes the liquid sheet, the laser energy excites the molecules of Rhodamine 6G so that the molecules can't remain in their vibrational energy states and move corresponding to the higher energy states and emit light at higher wavelengths. This kind of emission of absorbed energy occurs at a peak value of $\sim 567 \mathrm{~nm}$ wavelengths from the higher energy states. The fluorescence band-pass filter of $560 \pm 10 \mathrm{~nm}$ wavelength was fitted in front of the camera lens to capture the peak emission from the liquid sheet.

The arrangement of simultaneous VLIF containing two cameras and cuboidal laser volume relative to the liquid sheet is schematically shown in Fig. 1 . The camera-1 is focused on the top surface of the continuous liquid sheet at a $100 \mathrm{~mm}$ radial distance from the nozzle axis. Its field of view (FOV), $170.6 \mathrm{~mm} \times 148.7 \mathrm{~mm}$, covers the transition of a continuous sheet into its breakup. There is a possibility that some part of the sheet remains in focus while the remaining is defocus due to a change in inclination of liquid sheet as it spreads radially. This inclination is described by an angle made by the liquid sheet with nozzle axis referred to as sheet ejection angle and denoted by $\varnothing$ (Fig. 1). It may also lead to projection error while postprocessing images in this way. Thus, to overcome such difficulty, the camera was oriented at an angle equals to liquid sheet ejection angle $(\varnothing)$ while picturing the top view. Such camera position was achieved by using a camera mount with the provision of $360^{\circ}$ azimuthal, $180^{\circ}$ back \& forth, and $180^{\circ}$ yaw and pitch directional movement.
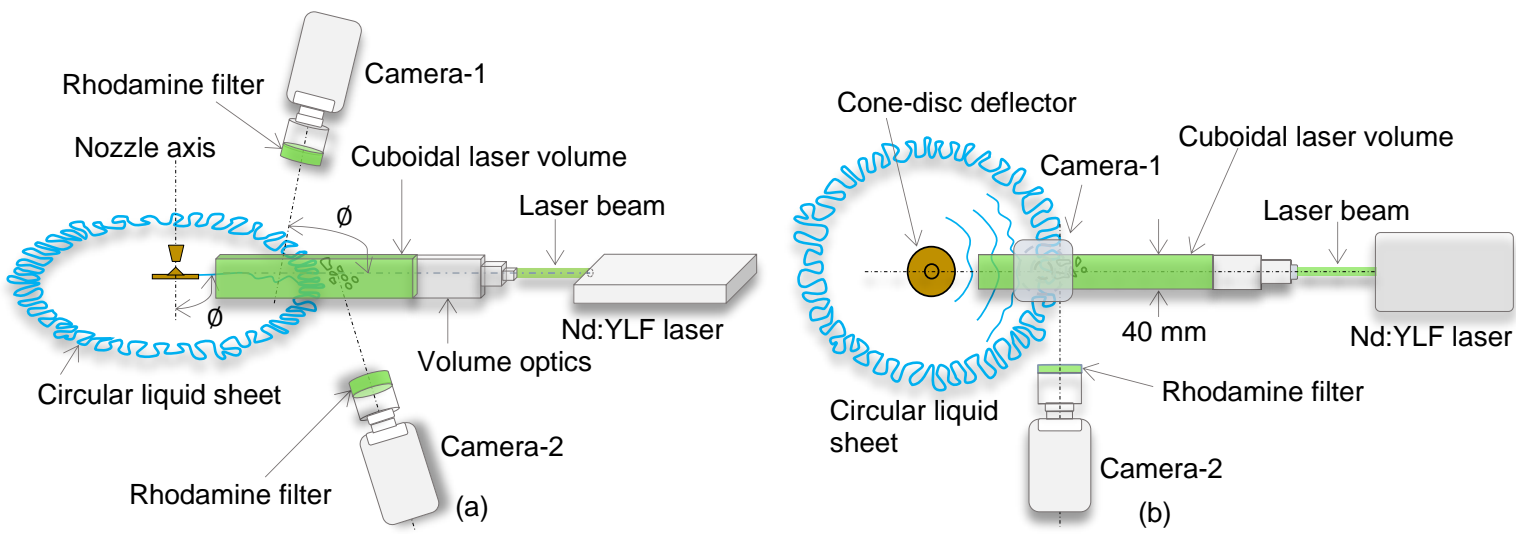

Figure 1. Schematic of simultaneous VLIF experimental arrangement in (a) isometric view and (b) plan view.

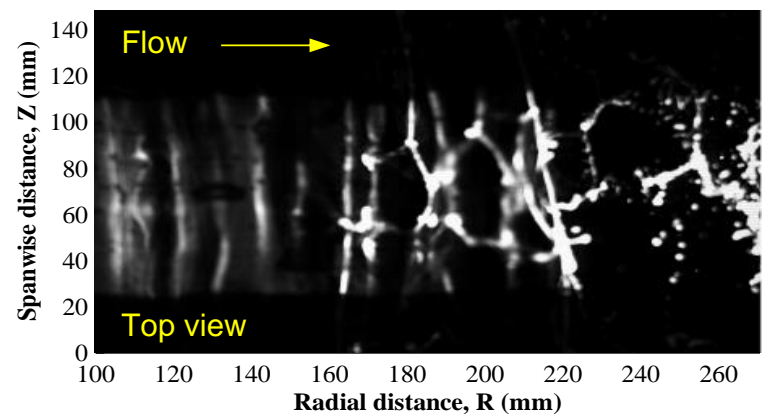

(a)

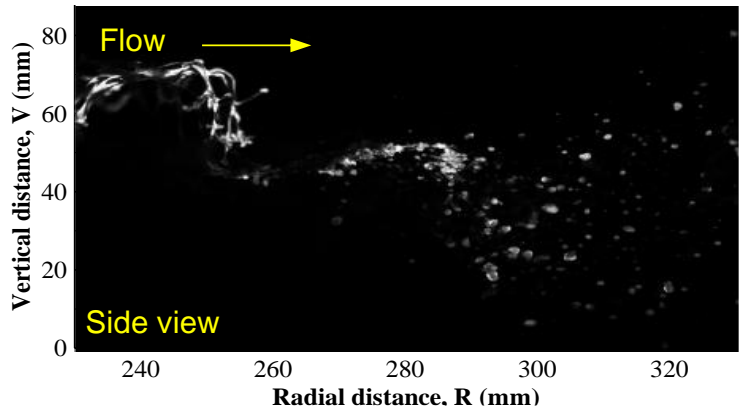

(b)

Figure 2. Instantaneous images of liquid sheet acquired respectively in (a) top and (b) side view VLIF at the same time instant and at $W e_{j e t}=1784$. 
Camera-2 is focused on the ligaments and droplets in a vertical plane of $40 \mathrm{~mm}$ width cuboidal laser volume (see plan view in Fig. 1b). The FOV of this camera is $100.3 \mathrm{~mm} \times 87.8 \mathrm{~mm}$ and at $230 \mathrm{~mm}$ radial location from nozzle axis. Calibration for both the FOVs was done with the help of a calibration plate to attain the scale factor, $f_{\text {scale }}(\mathrm{mm} /$ pixel). Figures $2(\mathrm{a})$ and $(\mathrm{b})$ are the instantaneous images of the actual flow field acquired at $6 \mathrm{kHz}$ framing rate and at the same time instant for $W e_{j e t}=1784$. The comprehensive details on simultaneous VLIF experiments are available in [15].

\section{Results and Discussion}

The top view and side view high-speed VLIF was simultaneously carried out to image various events involved in the breakup dynamics liquid sheet. The data were obtained by varying the liquid jet Weber number over a range of $996<W e_{j e t}<7480$. Liquid sheet was observed to be smooth at low jet Weber number $\left(W e_{j e t}\right)$. While the sheet is becoming unstable with an increase in $W e_{j e t}$. The present study aided the visualization of various phenomena that change spatiotemporally on the liquid sheet with $W e_{j e t}$. Various features are identified through observations made, such as growth of radial waves, formation of punctures in the liquid sheet, rim (front edge) breakup, coalescence of two perforations forming a liquid bridge, ligaments and droplets breakup, and other events leading to sheet breakup. It is challenging to pick which event is most responsible for sheet breakup. The present study focuses on identifying the dominant features leading to the sheet breakup using the POD tool.

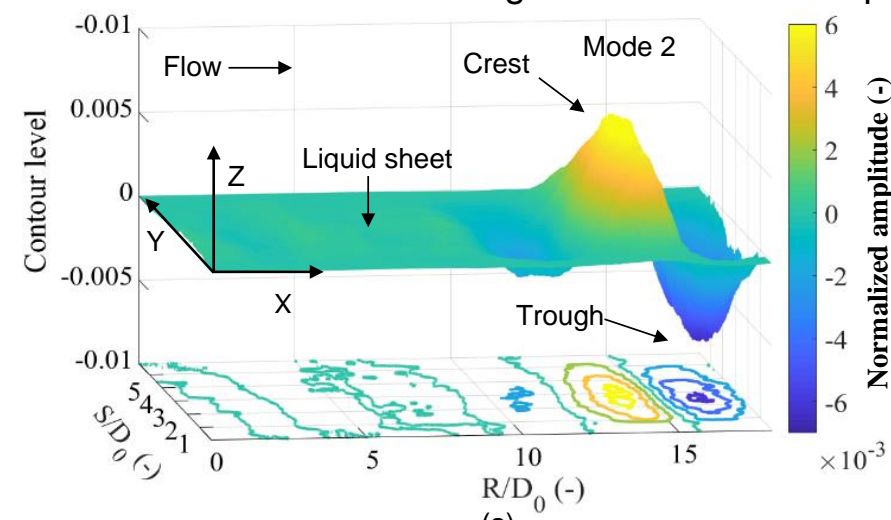

(a)

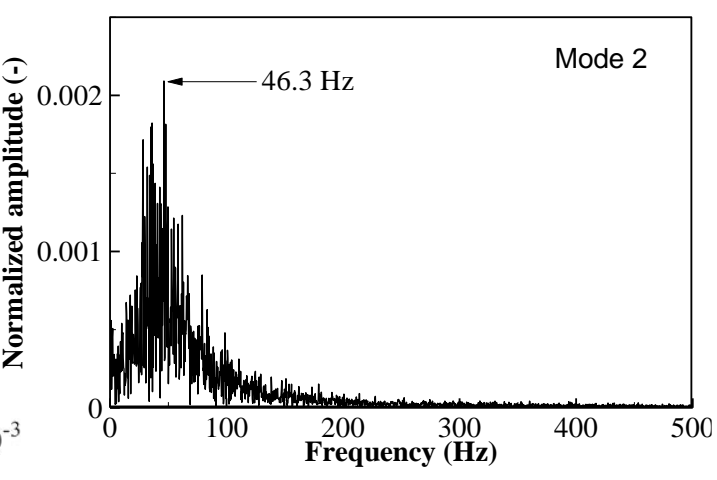

(b)

Figure 3. (a) Isometric view of $2^{\text {nd }}$ spatial POD mode and (b) corresponding temporal POD mode showing most dominant frequency of $46.3 \mathrm{~Hz}$ obtained by postprocessing top view VLIF at $W e_{j e t}=2484$. The light and dark contour levels represent crests and troughs of radial waves on a liquid sheet.

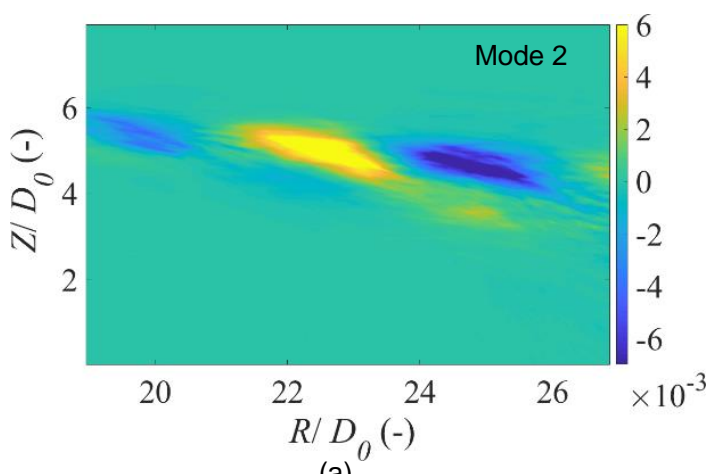

(a)

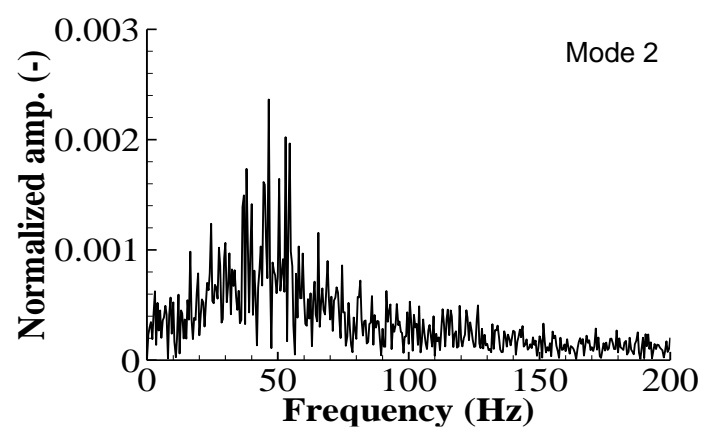

(b)

Figure 4. (a) $2^{\text {nd }}$ spatial POD mode and (b) corresponding temporal POD mode obtained by postprocessing side view VLIF at $W e_{j e t}=2484$. Light and dark contour levels represent crests and troughs of radial waves on a liquid sheet. 
The spatial and temporal POD modes are obtained for all flow conditions to investigate the variation in flow structures (or physical phenomena) and determine whether their dominant order changes with variation in inlet flow conditions. The first mode contains the highest energy in the energy distribution and is associated with the time-averaged mean of the entire data set. It is referred to as mean mode. The second energy mode represents a feature in the intensity field responsible for dominant variations superimposed on the mean mode. Similarly, other energy modes are arranged in descending order correspond to the principal phenomenon of flow field based on their contribution to the intensity field. Figure 3 demonstrating the $2^{\text {nd }}$ spatial and temporal POD modes for the flow field of top view VLIF at $W e_{j e t}=2484$. Similarly, the $2^{\text {nd }}$ spatial and temporal POD modes obtained from side view VLIF are shown in Fig. 4. It is to be noted that the temporal mode represented here are obtained after performing fast Fourier transform (FFT).

The vertical motion of expanding liquid sheet becomes rapid in a radially outward direction [14]. This motion can be verified by finding the liquid sheet oscillation frequencies at different radial locations. Figure 5 shows the variation of sheet oscillation frequency with $W e_{j e t}$. As expected, the oscillation of liquid sheet increases with an increase in jet Weber number $\left(W e_{j e t}\right)$. From the Fig. 5 , it is interesting to note that the sheet oscillation is dominant phenomenon for $W e_{j e t}<1500$.

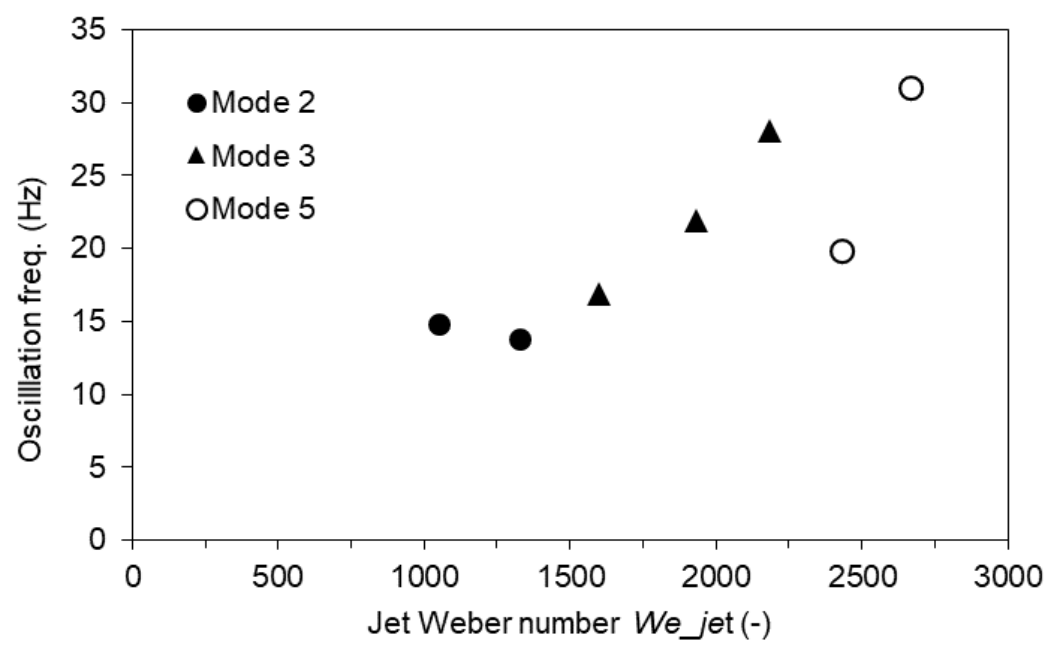

Figure 5. Temporal POD spectrum showing liquid sheet oscillation for various jet Weber numbers $\left(W e_{j e t}\right)$.

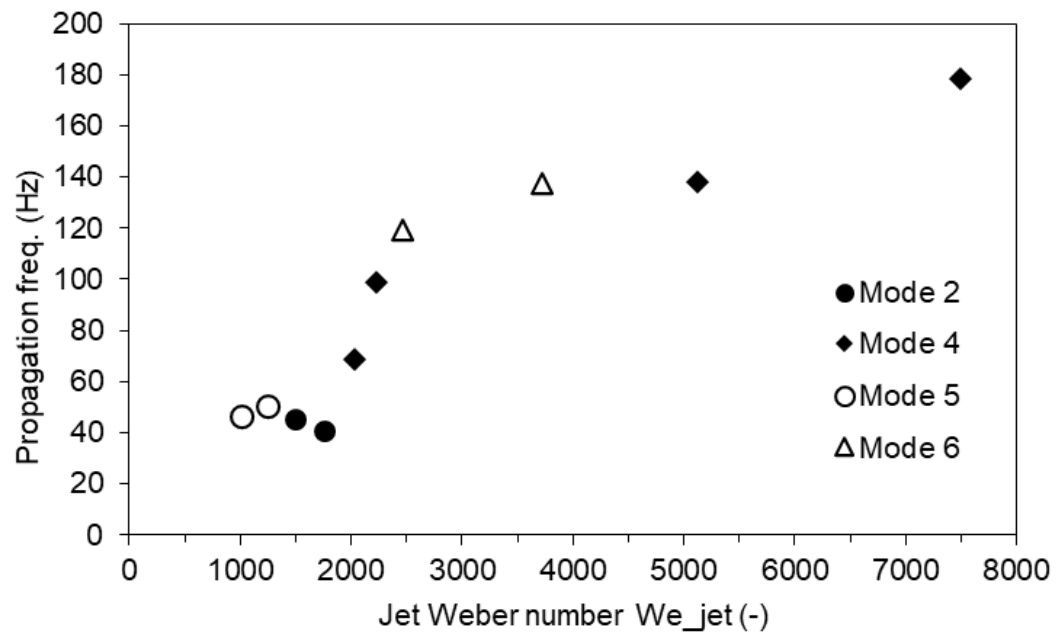

Figure 6. Initial temporal POD modes showing the frequency of propagation of radial waves on the liquid sheet for various inlet flow conditions or $W e_{j e t}$. 
The perturbations that are originating from the lip of the deflector disc and grow spatiotemporally are leading to sheet breakup due to Kelvin-Helmholtz instability [16-18]. Such a radial wave motion is not primarily a dominant flow structure compared to sheet oscillation and breakup, as noted from the side view VLIF imaging data. It appears in higherorder spatial POD modes. The frequency associated with the radial wave propagation for various $W e_{j e t}$ are shown in Fig. 6 . The overall trend shows that the propagation of crests or trough of radial waves increase with an increase in $W e_{j e t}$. The propagation frequencies are basically relevant to the phase velocity of radial waves on liquid sheet. The most dominant frequencies associated with the propagation are between the $W e_{j e t}$ range 1500-2000.

Liquid sheet breakup phenomenon is discussed next. It is observed from the high-speed imaging data that the liquid sheet breaks up at the outer edge at a specific time interval. The sheet breakup frequencies obtained from temporal POD modes for various flow conditions are shown in Fig. 7. The sheet breaks up more rapidly for higher $W e_{j e t}$. One can note this in Fig. 7 , where the solid black circles are representing the second temporal POD mode for $W e_{j e t} \geq$ 2000. As explained earlier, the first mode is the mean of entire flow field and the second mode is the most dominant mode for that flow field. Therefore, for $W e_{j e t} \geq 2000$, liquid sheet breakup is the most dominant phenomenon. Also, the breakup frequency increase with an increase in $W e_{j e t}$. It is to be noted that liquid sheet breakup is the foremost phenomenon dominating the oscillation and propagation for many flow conditions. One can also note that for lower jet Weber number $\left(W e_{j e t}<1500\right)$, the breakup is the second most dominant phenomenon. As mentioned above, the most dominant phenomenon for $W e_{j e t}<1500$ is liquid sheet oscillation.

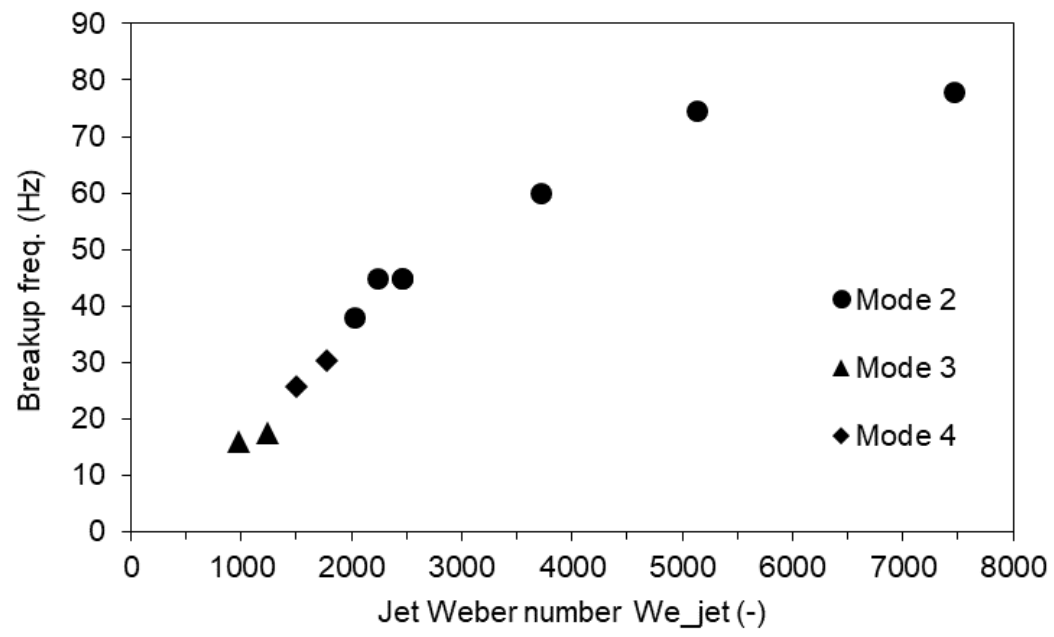

Figure 7. Breakup frequency of outer edge of liquid sheet representing initial temporal POD modes for various jet Weber numbers.

\section{Conclusions}

The simultaneous VLIF allowed the observation of various spatiotemporal flow features involved in sheet breakup. Advanced feature extraction and transient analysis method like proper orthogonal decomposition (POD) was applied to study the dominant flow features associated with sheet dynamics. Such events and their variation with inlet flow conditions are studied for the first time using the POD tool. The results of POD analysis showed sheet that the sheet oscillation is the most dominant phenomenon in the flow field for $W e_{j e t}<1500$. While the radial wave propagation in the streamwise direction is found to be the most dominant feature for $1500 \leq W e_{j e t}<2000$. For higher jet Weber number, $W e_{j e t} \geq 2000$, the liquid sheet breakup at the outer edge is the most dominant amongst all. 


\section{References}

1. Lefebvre, A.H.; McDonell, V.G. Atomization and Sprays; CRC Press, 2017; ISBN 9781315120911.

2. Lumley, J.L. Stochastic tools in turbulence. Appl. Math. Mech. 1970, 12, 194.

3. Chatterjee, A. An introduction to the proper orthogonal decomposition. Curr. Sci. 2004, 78.

4. $\quad$ Liang, Y.C.; Lee, H.P.; Lim, S.P.; Lin, W.Z.; Lee, K.H.; Wu, C.G. Proper orthogonal decomposition and its applications - Part I: Theory. J. Sound Vib. 2002, 252, 527544, doi:10.1006/jsvi.2001.4041.

5. $\quad$ Liang, Y.C.; Lee, H.P.; Lim, S.P.; Lin, W.Z.; Lee, K.H.; Wu, C.G. Proper Orthogonal Decomposition and Its Applications \} Part li : Model Reduction for Mems Dynamical Analysis. J. Sound Vib. 2002, 256, doi:10.1006/jsvi.5007.

6. Berkooz, G.; Holmes, P.; Lumley, J.L. The proper orthogonal decomposition in the analysis of turbulent flows. Annu. Rev. Fluid Mech. 1993, 25, 539-575.

7. Arienti, M.; Soteriou, M.C. Time-resolved proper orthogonal decomposition of liquid jet dynamics. Phys. Fluids 2009, 21, 1-15, doi:10.1063/1.3263165.

8. Bonnet, J.P.; Cole, D.R.; Delville, J.; Glauser, M.N.; Ukeiley, L.S. Stochastic estimation and proper orthogonal decomposition: Complementary techniques for identifying structure. Exp. Fluids 1994, 17, 307-314, doi:10.1007/BF01874409.

9. Chatterjee, S.; Das, M.; Mukhopadhyay, A.; Sen, S. Experimental Investigation of Breakup of Annular Liquid Sheet in a Hybrid Atomizer. J. Propuls. Power 2015, 31, 1232-1241, doi:10.2514/1.B35574.

10. Vegad, C.S.; Kumar, A.; Chakravarthy, S.R. Experimental study of a free-surface circular liquid sheet using planar laser-induced fluorescence. Exp. Fluids 2020, 61, 46, doi:10.1007/s00348-020-2881-4.

11. Zhou, X.; Yu, H.Z. Experimental investigation of spray formation as affected by sprinkler geometry. Fire Saf. J. 2011, 46, 140-150, doi:10.1016/j.firesaf.2011.01.003.

12. Haynes, W.M.; Lide, D.R.; Bruno, T.J. CRC Handbook of Chemistry and Physics; Haynes, W.M., Lide, D.R., Bruno, T.J., Eds.; 93rd ed.; CRC Press, Taylor and Francis Group: Boca Raton, Londaon, New York, 2012; ISBN 978-1-4398-8050-0.

13. Vargaftik, N.B.; Volkov, B.N.; Voljak, L.D. International tables of the surface tension of water. J. Phys. Chem. Ref. Data 1983, 12, 817-820, doi:10.1063/1.555688.

14. Vegad, C.S.; Chakravarthy, S.R.; Kumar, A. Dynamics of a radially expanding circular liquid sheet and its atomization characteristics. Fire Saf. J. 2018, 100, 51-63, doi:10.1016/j.firesaf.2018.07.004.

15. Vegad, C.S.; Kumar, A.; Chakravarthy, S.R. Visualization of features of atomizing circular liquid sheet using simultaneous Volume Laser-Induced Fluorescence. Exp. Therm. Fluid Sci. 2020, 112, 109947, doi:10.1016/j.expthermflusci.2019.109947.

16. Taylor, G. Formation of thin flat sheets of water. Proc. R. Soc. London. Ser. A. Math. Phys. Sci. 1961, 259, 1-17, doi:10.1098/rspa.1960.0207.

17. Lin, S.P. Breakup of liquid sheets and jets; Cambridge University Press, 2003; Vol. 39; ISBN 9780521806947.

18. Huang The breakup of axisymmetric liquid sheets. J. Fluid Mech. 1970, 43, 305, doi:10.1017/S0022112070002392. 